JOURNAL OF INTEGRAL EQUATIONS

AND APPLICATIONS

Volume 7, Number 4, Fall 1995

\title{
REDUCTION OF AN INFINITE SYSTEM OF INTEGRODIFFERENTIAL EQUATIONS FOR ELECTRIC CURRENTS ON A LATTICE OF CLOSED CURVES TO A FINITE SYSTEM OF INDEPENDENT PSEUDODIFFERENTIAL EQUATIONS ON A CIRCLE
}

\author{
VALERY A. KHOLODNYI
}

\begin{abstract}
An infinite system of integrodifferential equations for electric currents on an infinite network of nonintersecting infinitely smooth simple closed curves in the plane is considered. These curves are obtained from a fixed curve by a parallel translation by vectors belonging to a one-dimensional lattice. An incident electromagnetic field is assumed to be harmonically time dependent and to be normally polarized so that the electric currents flow only along the curves. The medium is assumed to be linear and isotropic. It is shown that if the incident electromagnetic field is a $T$-periodic function on the lattice, then the original system can be reduced to a system of $T$ independent pseudodifferential equations on the unit circle with classic elliptic pseudodifferential operators of order 1 in the Sobolev scale. Another significant outcome of this work is that this reduction allows one to apply the many known powerful methods for the numerical analysis of classic elliptic pseudodifferential equations on the unit circle to the original system.
\end{abstract}

1. Introduction. It is well known (see, for example, $[\mathbf{1}, \mathbf{2}, \mathbf{3}]$ ) that boundary-value problems for Maxwell's equations in a plane isotropic linear medium in which the boundary consists of a finite number $N$ of conducting nonintersecting infinitely smooth simple closed curves can be reduced to a system of $N$ integrodifferential equations for electric currents along the curves. Harmonic time dependency, normal polarization of the electromagnetic field (i.e., that the vector of electric field strength lies in the plane), and the radiation condition at infinity were assumed. The $N \times N$ matrix integrodifferential operator of the system was proven to be an $N \times N$ matrix classic elliptic pseudodifferential operator of order 1 in the Sobolev scale of $N$-dimensional

Received by the editors on March 17, 1995, and in revised form on July 18, 1995.

1991 Mathematics Subject Classification. Primary: 45J05, 45F15, 47G30, Secondary: 35Q60, 78A45, 78A50.

Key words and phrases. Systems of integrodifferential equations, pseudodifferential equations, Maxwell's equations in periodic structures.

Copyright (C)1995 Rocky Mountain Mathematics Consortium 
complex vector functions on the unit circle. The practical benefit of viewing the original system of integrodifferential equations as a system of pseudodifferential equations is that one can then apply the many known powerful methods for the numerical analysis of such systems of pseudodifferential equations (see, for example, $[\mathbf{4 - 6 , 9}]$ and references therein).

In the present paper we consider a particular boundary formed not by a finite, but by an infinite number of such curves. We follow the approach introduced in [7], where we applied the discrete Fourier transform to reduce an infinite system of integral equations on this particular boundary to a finite system of independent pseudodifferential equations on the unit circle with classic elliptic pseudodifferential operators of order -1 in the Sobolev scale. (This infinite system of integral equations arises in the reduction of the Dirichlet problem for the Helmholtz equation in the plane to this particular boundary.) Here we develop this approach, applying the discrete Fourier transform to reduce an infinite system of integrodifferential equations for electric currents along the curves forming this particular boundary to a finite system of independent pseudodifferential equations on the unit circle with classic elliptic pseudodifferential operators, but of order 1 in the Sobolev scale.

Let $\gamma$ be an infinitely smooth, simple closed curve in the plane with coordinates $\left(x_{1}, x_{2}\right)$. Without loss of generality, we assume the length of $\gamma$ to be equal to $2 \pi$. Let $\bar{x}(\xi)=\left(x_{1}(\xi), x_{2}(\xi)\right)$ define the curve $\gamma$ parametrically where $\xi$ is the natural parameter on $\gamma$. It is obvious that $\bar{x}(\xi)$ is an infinitely smooth function. We denote by $\Gamma$ the following infinite network of curves in the plane:

$$
\Gamma=\left\{\gamma_{m}: \bar{x}_{m}(\xi)=\bar{x}(\xi)+\bar{h} m, m \in \mathbf{Z}, \xi \in[0 ; 2 \pi]\right\},
$$

where $\bar{h}$ is the vector $(h, 0)$ with $h>\sup \left\{\left|x_{1}(\xi)-x_{1}(\eta)\right|: \xi, \eta \in[0,2 \pi]\right\}$, $\bar{x}_{m}(\xi)$ defines curve $\gamma_{m}$ parametrically, and $\mathbf{Z}$ is the set of integers.

Remark. Since $\gamma$ is a closed curve, $\sup \left\{\left|x_{1}(\xi)-x_{1}(\eta)\right|: \xi, \eta \in[0,2 \pi]\right\}$ is finite. This supremum is chosen as a lower bound for $h$ to ensure that no two curves from $\Gamma$ intersect. Then $\Gamma$ is an infinite set of nonintersecting identical curves $\gamma_{m}$ obtained from a fixed curve $\gamma$ by a parallel translation by vectors $\bar{h} m, m \in \mathbf{Z}$, which form a onedimensional lattice with a lattice constant $h$. 
Each curve $\gamma_{m}$ from the network $\Gamma$ physically plays either the role of the cross-section of a hollow cylindrical conductor or the boundary of the cross-section of a solid cylindrical conductor that is orthogonal to the plane. We assume that all curves $\gamma_{m}$ have the same surface conductivity $\sigma$. For the sake of generality we allow $\sigma$ to vary over $\xi$. More precisely, we assume $\sigma=\sigma(\xi)$ to be an infinitely smooth complex function of $\xi$ on $\gamma$. According to the physical meaning, $\sigma(\xi) \neq 0$ for all $\xi$ on $\gamma$. Also we consider the case of perfect conductors formally described by $\sigma=\infty$. The network of curves $\Gamma$ is surrounded by a medium which we assume to be linear and isotropic with dielectric constant $\varepsilon>0$ and permeability $\mu>0$. The electromagnetic field is assumed to be harmonically time-dependent and to be normally polarized so that the electric currents flow only along the curves.

We will treat a complex function on a curve $\gamma_{m}$ in $\Gamma$ as a complex function on the unit circle $S$, and we will identify it with the corresponding $2 \pi$-periodic function on the real line.

The infinite system of integrodifferential equations for the complex amplitudes of electric current densities $I_{l}$ along $\gamma_{l}$ in $\Gamma$ (see the Appendix for the derivation) can be shown to be:

$$
\sum_{l=-\infty}^{\infty} \hat{Z}_{m-l} I_{l}=E_{m}, \quad m \in \mathbf{Z}
$$

where $E_{m}=E_{m}(\xi)$ is the complex amplitude of the tangential component of an incident electric field on $\gamma_{m}$ at the point $\xi$, and where the operator $\hat{Z}_{m-l}$ is defined by

$$
\hat{Z}_{m-l}=\frac{i \omega}{c^{2}} \hat{L}_{m-l}+\hat{R}_{m-l}+\frac{1}{i \omega} \frac{\hat{1}}{C_{m-l}},
$$

with operators $\hat{L}_{m-l}, \hat{R}_{m-l}$ and $\left(\hat{1} / C_{m-l}\right)$ given by

$$
\begin{aligned}
&\left(\hat{L}_{m-l} I_{l}\right)(\xi)=\mu \frac{i}{4} \int_{0}^{2 \pi}\langle\tau(\xi) ; \tau(\eta)\rangle H_{0}^{(2)}(k \mid \bar{x}(\xi) \\
&-\bar{x}(\eta)+\bar{h}(m-l) \mid) I_{l}(\eta) d \eta \\
&\left(\hat{R}_{m-l} I_{l}\right)(\xi)=\delta(m-l) \sigma^{-1}(\xi) I_{l}(\xi) \\
&\left(\frac{\hat{1}}{C_{m-l}} I_{l}\right)(\xi)=-\frac{1}{\varepsilon} \frac{i}{4} \frac{d}{d \xi} \int_{0}^{2 \pi} H_{0}^{(2)}(k \mid \bar{x}(\xi) \\
&-\bar{x}(\eta)+\bar{h}(m-l) \mid) \frac{d}{d \eta} I_{l}(\eta) d \eta
\end{aligned}
$$


Here $c$ is the speed of light in a vacuum, $\omega>0$ is the angular frequency, $k=\sqrt{\varepsilon \mu}(\omega / c)$ is the wave number, $\langle;\rangle$ and $|\cdot|$ stand for the standard scalar product and the Cartesian distance in the plane, $\tau(\xi)=\left((\partial / \partial \xi) x_{1}(\xi),(\partial / \partial \xi) x_{2}(\xi)\right)$ is a unit vector tangent to the curve $\gamma$ at the point $\xi, H_{0}^{(2)}$ is the Hankel function, and $\delta(n)$ is the Kronecker symbol.

Remark. In the case of perfect conductors, $\hat{R}_{m-l}$ is the identically zero operator for all $m$ and $l$ in $\mathbf{Z}$.

Remark. The problem of determining such electric current densities on the network of curves $\Gamma$ arises in various areas of radio physics and electrical engineering including diffraction, antenna theory, wave propagation in periodic structures and many others (see, for example, [11 and 12]).

Remark. The operators $\hat{Z}_{m-l}, \hat{L}_{m-l}, \hat{R}_{m-l}$ and $\left(\hat{1} / C_{m-l}\right)$ have the physical meaning of mutual (self, if $m=l$ ) operator impedance, inductance, resistance and inverse capacitance of the conductors with surfaces $\gamma_{m}$ and $\gamma_{l}$.

We recall [1] that the Sobolev space $H_{t}=H_{t}(S)$ of complex functions on $S$ is the completion of the space $C^{\infty}(S)$ of infinitely smooth complex functions on $S$ with respect to the norm

$$
\|u\|_{t}=\left(\sum_{p=-\infty}^{\infty}\left(1+p^{2}\right)^{t}\left|C_{p}(u)\right|^{2}\right)^{1 / 2},
$$

where $C_{p}(u)$ are the Fourier coefficients of the function $u$ with respect to the system $\left\{e^{i p \xi}, p \in \mathbf{Z}\right\}$. The spaces $H_{t}, t$ in $\mathbf{R}$ (the set of reals), form the Banach scale.

Also we recall that if $g$ is an $N$-periodic map from a set of positive integers $1,2, \ldots, N$ into a Banach space, then the discrete Fourier transform of $g$ is defined by

$$
\hat{g}_{s}=\left(F_{m \rightarrow s} g\right)_{s}=\sum_{m=1}^{N} e^{-i(2 \pi / N) s m} g_{m},
$$


where $\hat{g}$ stands for the discrete Fourier image of $g$.

The inverse discrete Fourier transform is given by

$$
g_{m}=\left(F_{s \rightarrow m}^{-1} \hat{g}\right)_{m}=\frac{1}{N} \sum_{s=1}^{N} e^{i(2 \pi / N) m s} \hat{g}_{s} .
$$

Hereafter, we will reserve the subscript $s$ to denote the image $g_{s}$ of the discrete Fourier transform of $g$, omitting the hat.

Let us formulate the following conditions:

Condition 1 (Periodicity). There exists a positive integer $T$ such that $E_{m+T}=E_{m}$ and $I_{m+T}=I_{m}$ for each $m$ in $\mathbf{Z}$. Hereafter, $T$ will stand for the least such integer.

Condition 2 (Nonresonance). The product $k h T \neq 0 \bmod 2 \pi$.

Remark. Our periodicity condition is a very natural one and holds in numerous applications dealing with electromagnetic wave propagation, that is, with electromagnetic processes periodic with respect to the network of curves $\Gamma$.

Remark. The $T$-periodicity of the function $E_{m}$ on the lattice corresponds to the $T h$-periodicity of the incident electric field in the $x_{1}$ direction in the plane. Therefore, the nonresonance condition means that the electromagnetic wave length of free space $\lambda=2 \pi / k$ does not fit into the distance $T h$ an integral number of times.

We denote by $\hat{\mathcal{Z}}_{s}$ the following operator:

$$
\hat{\mathcal{Z}}_{s}=\sum_{l=1}^{T} e^{-i(2 \pi / T) s l} \hat{\mathcal{Z}}_{l}, \quad s=1, \ldots, T,
$$

where the operator $\hat{\mathcal{Z}}_{l}$ is given by:

$$
\hat{\mathcal{Z}}_{l}=\sum_{v=-\infty}^{\infty} \hat{Z}_{l+v T}, \quad l \in \mathbf{Z} .
$$


We consider the following system of $T$ independent equations in the unknown functions $I_{s}$ :

$$
\hat{\mathcal{Z}}_{s} I_{s}=E_{s}, \quad s=1, \ldots, T,
$$

where the functions $E_{s}$ are given by

$$
E_{s}=\sum_{m=1}^{T} e^{-i(2 \pi / T) s m} E_{m} .
$$

We are now ready to present the main result of this paper.

Theorem. Let Conditions 1 and 2 hold, and let $E_{m}$ belong to $H_{t}$ for all $m=1, \ldots, T$. Then

i) Systems (1) and (6) are equivalent in the sense that their solutions $I_{l}$ and $I_{s}$ are the discrete Fourier images of each other:

$$
I_{l}=\frac{1}{T} \sum_{s=1}^{T} e^{i(2 \pi / T) l s} I_{s}
$$

ii) The operators $\hat{\mathcal{Z}}_{s}$ for all $s=1, \ldots, T$ are classic elliptic pseudodifferential operators of order 1 in the Sobolev scale on $S$;

iii) All solutions $I_{l}$ of system (1) belong to $H_{t+1}$ for all $l=1, \ldots, T$.

Remark. Under Conditions (1) and (2) the Theorem reduces the problem of solving system (1) to that of system (6) which consists of $T$ independent pseudodifferential equations on the unit circle with classic elliptic pseudodifferential operators of order 1 in the Sobolev scale. Also since the kernel subspaces of such pseudodifferential operators are at most finite dimensional $[\mathbf{1}]$, the operators $\hat{\mathcal{Z}}_{s}$, for all $s=1, \ldots, T$, are invertible except at most on some finite dimensional subspaces.

2. Proof of the theorem. In order to prove the theorem we need some preparation. For convenience of reference, we present here Lemma 1 introduced and proven by the author in $[7]$. 
Lemma 1. The function

$$
G_{l}(\xi, \eta)=\sum_{\substack{v=-\infty \\ v: l+v T \neq 0}}^{\infty} H_{0}^{(2)}(k|\bar{x}(\xi)-\bar{x}(\eta)+\bar{h}(l+v T)|)
$$

is infinitely smooth on the torus $S \times S$ whenever $l \in \mathbf{Z}$ and $k h T \neq$ $0 \bmod 2 \pi$.

For each $l \in \mathbf{Z}$ we consider the following sequences of operators:

$$
\begin{gathered}
\hat{L}_{l}^{n}=\sum_{|v| \leq n} \hat{L}_{l+v T}, \quad \hat{R}_{l}^{n}=\sum_{|v| \leq n} \hat{R}_{l+v T} \\
\frac{\hat{1}^{n}}{C_{l}}=\sum_{|v| \leq n} \frac{\hat{1}}{C_{l+v T}},
\end{gathered}
$$

where $n \in \mathbf{Z}_{+}$, the set of nonnegative integers.

We denote by $\|\cdot\|_{\left(t_{1} \rightarrow t_{2}\right)}$ the norm in the Banach space $O_{\left(t_{1} \rightarrow t_{2}\right)}$ of bounded linear operators acting from $H_{t_{1}}$ to $H_{t_{2}}$.

In what follows, whenever we refer to the order of an operator we mean the order in the Sobolev scale on $S$.

Lemma 2. Let Condition 2 hold. Then there exist such operators $\hat{\mathcal{L}}_{l}, \hat{\mathcal{R}}_{l}$ and $\left(\hat{1} / \mathcal{C}_{l}\right)$ that

i) $\hat{\mathcal{L}}_{l}, \hat{\mathcal{R}}_{l}$ and $\left(\hat{1} / \mathcal{C}_{l}\right)$ are classic elliptic pseudodifferential operators (PDO) of orders $-1,0$ and 1 , respectively, if $l=0 \bmod T$ and are operators of order $-\infty$ if $l \neq 0 \bmod T$. In the case of perfect conductors, $\hat{\mathcal{R}}_{l}$ is the identically zero operator for all $l$ in $\mathbf{Z}$;

ii) For each $l \in \mathbf{Z}$ and each $t \in \mathbf{R}$, as $n \rightarrow \infty$, we have

$$
\begin{gathered}
\left\|\hat{\mathcal{L}}_{l}-\hat{L}_{l}^{n}\right\|_{(t \rightarrow t+1)} \rightarrow 0, \quad\left\|\hat{\mathcal{R}}_{l}-\hat{R}_{l}^{n}\right\|_{(t \rightarrow t)} \rightarrow 0 \\
\left\|\frac{\hat{1}}{\mathcal{C}_{l}}-\frac{\hat{1}^{n}}{C_{l}}\right\|_{(t \rightarrow t-1)} \rightarrow 0 ;
\end{gathered}
$$

iii) For each $l \in \mathbf{Z}$,

$$
\hat{\mathcal{L}}_{l+T}=\hat{\mathcal{L}}_{l}, \quad \hat{\mathcal{R}}_{l+T}=\hat{\mathcal{R}}_{l}, \quad \frac{\hat{1}}{\mathcal{C}_{l+T}}=\frac{\hat{1}}{\mathcal{C}_{l}} .
$$


Proof. We prove Lemma 2 for the operators $\hat{\mathcal{L}}_{l}, \hat{\mathcal{R}}_{l}$ and $\left(\hat{1} / \mathcal{C}_{l}\right)$ separately. Let us consider an operator $\hat{\mathcal{L}}_{l}$ with the kernel

$$
\mu\langle\tau(\xi) ; \tau(\eta)\rangle K_{l}(\xi, \eta), \quad l \in \mathbf{Z},
$$

where

$$
K_{l}(\xi, \eta)=\frac{i}{4} \sum_{v=-\infty}^{\infty} H_{0}^{(2)}(k|\bar{x}(\xi)-\bar{x}(\eta)+\bar{h}(l+v T)|) .
$$

We are going to show that the operator $\hat{\mathcal{L}}_{l}$ satisfies (i), (ii) and (iii). To prove (i), we split the kernel (7) into two summands:

$$
\mu\langle\tau(\xi) ; \tau(\eta)\rangle\left(K_{0}(\xi, \eta)+K_{l}^{\prime}(\xi, \eta)\right),
$$

where

$$
\begin{aligned}
K_{0}(\xi, \eta) & =\frac{i}{4} H_{0}^{(2)}(k|\bar{x}(\xi)-\bar{x}(\eta)|) \sum_{v=-\infty}^{\infty} \delta(l+v T), \\
K_{l}^{\prime}(\xi, \eta) & =\frac{i}{4} \sum_{\substack{v=-\infty \\
v: l+v T \neq 0}}^{\infty} H_{0}^{(2)}(k|\bar{x}(\xi)-\bar{x}(\eta)+\bar{h}(l+v T)|) .
\end{aligned}
$$

By Lemma 1 the function $K_{l}^{\prime}(\xi, \eta)$ belongs to $C^{\infty}(S \times S)$, the space of infinitely smooth complex functions on the torus $S \times S$ with the standard topology, for every $l \in \mathbf{Z}$. Therefore, using the definition of the Hankel function [8], we can rewrite the kernel (7) as follows:

$$
\begin{aligned}
\mu \frac{1}{2 \pi}\langle\tau(\xi) ; \tau(\eta)\rangle J_{0}(k \mid \bar{x}(\xi)- & \bar{x}(\eta) \mid) \ln (k \mid \bar{x}(\xi) \\
& -\bar{x}(\eta) \mid) \sum_{v=-\infty}^{\infty} \delta(l+v T)+g_{l}(\xi, \eta)
\end{aligned}
$$

where $g_{l}(\xi, \eta)$ is in $C^{\infty}(S \times S)$ for every $l \in \mathbf{Z}$, and $J_{0}$ is the Bessel function. By construction of the vector $\tau(\xi)$ and by the definition of the Bessel function $J_{0}[\mathbf{8}]$, the product $\left.\langle\tau(\xi) ; \tau(\eta)\rangle J_{0}(k|\bar{x}(\xi)-\bar{x}(\eta)|)\right|_{\eta=\xi}$ is not zero (it is equal to 1 ) for all $\xi \in S$. Therefore, according to [1] the 
kernel (10) defines a classic elliptic PDO of order -1 if $l=0 \bmod T$ and an operator of order $-\infty$ if $l \neq 0 \bmod T$.

Let us prove (ii). Consider the kernel of the operator $\Delta \hat{\mathcal{L}}_{l}^{n}=\hat{\mathcal{L}}_{l}-\hat{L}_{l}^{n}$ given for $n>|l / T|$ by

$$
\mu \frac{i}{4}\langle\tau(\xi) ; \tau(\eta)\rangle \sum_{|v|>n} H_{0}^{(2)}(k|\bar{x}(\xi)-\bar{x}(\eta)+\bar{h}(l+v T)|) .
$$

To prove (ii), it is enough to show that, if $l \in \mathbf{Z}$ and $k h T \neq 0 \bmod 2 \pi$, the above kernel converges to the zero function in the topology of $C^{\infty}(S \times S)$ as $n \rightarrow \infty$. But this follows directly from Lemma 1 .

Statement (iii) follows immediately from the explicit form of the kernel of the operator $\hat{\mathcal{L}}_{l}$ given by expressions (7) and (8).

Let us notice first that in the case of perfect conductors, since $\hat{R}_{m-l}$ is the identically zero operator for all $m$ and $l$ in $\mathbf{Z}$, the identically zero operator $\hat{\mathcal{R}}_{l}, l \in \mathbf{Z}$, satisfies (ii) and (iii). Now let us consider the operator $\hat{\mathcal{R}}_{l}$ of multiplication by the function

$$
\sigma^{-1}(\xi) \sum_{v=-\infty}^{\infty} \delta(l+v T), \quad l \in \mathbf{Z}
$$

We are going to show that the operator $\hat{\mathcal{R}}_{l}$ satisfies (i), (ii) and (iii). To prove (i), notice that if $l=0 \bmod T$, then $\hat{\mathcal{R}}_{l}$ is the operator of multiplication by the function $\sigma^{-1}(\xi)$ in $C^{\infty}(S)$ where $\sigma^{-1}(\xi) \neq 0$ for any $\xi \in S$. Therefore, by [1], $\hat{\mathcal{R}}_{l}$ is a classic elliptic PDO of order 0 . If $l \neq 0 \bmod T$, then $\hat{\mathcal{R}}_{l}$ is the identically zero operator and, hence, by $[\mathbf{1}]$, it is an operator of order $-\infty$.

To prove (ii) notice that, if $l \neq 0 \bmod T$, the sequence $\hat{R}_{l}^{n}$ is the stationary sequence of the identically zero operators. If $l=0 \bmod T$, the sequence $\hat{R}_{l}^{n}$ for $n>|l / T|$ is the stationary sequence of the operators of multiplication by the function $\sigma^{-1}(\xi)$.

Statement (iii) immediately follows from the explicit form of the operator $\hat{\mathcal{R}}_{l}$ given by expression (11).

Finally, let us consider the operator

$$
\frac{\hat{1}}{\mathcal{C}_{l}}=-\frac{1}{\varepsilon} \frac{d}{d \xi} \mathcal{A}_{l} \frac{d}{d \xi}, \quad l \in \mathbf{Z},
$$


where the operator $\mathcal{A}_{l}$ has the kernel $K_{l}(\xi, \eta)$ defined by expression (8). We are going to show that the operator $\left(\hat{1} / \mathcal{C}_{l}\right)$ satisfies (i), (ii) and (iii). To prove (i), we split $K_{l}(\xi, \eta)$ into two summands as in expression (9) and represent the operator $\left(\hat{1} / \mathcal{C}_{l}\right)$ as follows

$$
\frac{\hat{1}}{\mathcal{C}_{l}}=-\frac{1}{\varepsilon}\left(\frac{d}{d \xi} \mathcal{A}_{0} \frac{d}{d \xi}+\frac{d}{d \xi} \mathcal{A}_{l}^{\prime} \frac{d}{d \xi}\right)
$$

where the operators $\mathcal{A}_{0}$ and $\mathcal{A}_{l}^{\prime}$ have the kernels $K_{0}(\xi, \eta)$ and $K_{l}^{\prime}(\xi, \eta)$, respectively. If $l=0 \bmod T$, then according to [1], the operator $\mathcal{A}_{0}$ is a classic elliptic PDO of order -1 , and hence the operator $(d / d \xi) \mathcal{A}_{0}(d / d \xi)$ as a composition of three classic elliptic PDOs of orders $1,-1$ and 1 , respectively, is a classic elliptic PDO of order 1 . Since $K_{l}^{\prime}(\xi, \eta)$ belongs to $C^{\infty}(S \times S)$ for every $l$ in $\mathbf{Z}, \mathcal{A}_{l}^{\prime}$ is an operator of order $-\infty$ and hence $(d / d \xi) \mathcal{A}_{l}^{\prime}(d / d \xi)$ is also an operator of order $-\infty$. Thus, if $l=0 \bmod T$, the operator $\left(\hat{1} / \mathcal{C}_{l}\right)$, as a sum of a classic elliptic PDO of order 1 and an operator of order $-\infty$, is a classic elliptic PDO of order 1 . If $l \neq 0 \bmod T, \mathcal{A}_{0}$ is the identically zero operator and hence $\left(\hat{1} / \mathcal{C}_{l}\right)$ is an operator of order $-\infty$.

Now let us prove (ii). Consider the operator $\Delta\left(\hat{1}^{n} / \mathcal{C}_{l}\right)=\left(\hat{1} / \mathcal{C}_{l}\right)-$ $\left(\hat{1}^{n} / C_{l}\right)$ given according to expression $(12)$ by $-(1 / \varepsilon)(d / d \xi) \Delta \mathcal{A}_{l}^{n}(d / d \xi)$ where the operator $\Delta \mathcal{A}_{l}^{n}$ has the following kernel for $n>|l / T|$ :

$$
\frac{i}{4} \sum_{|v|>n} H_{0}^{(2)}(k|\bar{x}(\xi)-\bar{x}(\eta)+\bar{h}(l+v T)|) .
$$

To prove (ii) it is enough to show that, if $l \in \mathbf{Z}$ and $k h T \neq 0 \bmod 2 \pi$, the above kernel converges to the zero function in the topology of $C^{\infty}(S \times S)$ as $n \rightarrow \infty$. But this follows directly from Lemma 1 .

Statement (iii) follows immediately from the explicit form of the operator $\left(\hat{1} / \mathcal{C}_{l}\right)$ given by expression $(12)$. This completes the proof of Lemma 2.

For each $l \in \mathbf{Z}$, consider the following sequence of operators

$$
\hat{Z}_{l}^{n}=\sum_{|v| \leq n} \hat{Z}_{l+v T}, \quad n \in \mathbf{Z}_{+} .
$$


Lemma 2, along with the definition of operators $\hat{Z}_{m-l}$ from expression (2), have the following obvious corollary:

Corollary. Let Condition 2 hold. Then the operator $\hat{\mathcal{Z}}_{l}$ defined in expression (5) has the following properties

i) $\hat{\mathcal{Z}}_{l}$ is a classic elliptic PDO of order 1 if $l=0 \bmod T$ and is an operator of order $-\infty$ if $l \neq 0 \bmod T$;

ii) For each $l \in \mathbf{Z}$ and $t \in \mathbf{R},\left\|\hat{\mathcal{Z}}_{l}-\hat{Z}_{l}^{n}\right\|_{(t \rightarrow t-1)} \rightarrow 0$ as $n \rightarrow \infty$;

iii) For each $l \in \mathbf{Z}, \hat{\mathcal{Z}}_{l+T}=\hat{\mathcal{Z}}_{l}$.

Remark. It is also obvious that

$$
\hat{\mathcal{Z}}_{l}=\frac{i \omega}{c^{2}} \hat{\mathcal{L}}_{l}+\hat{\mathcal{R}}_{l}+\frac{1}{i \omega} \frac{\hat{1}}{\mathcal{C}_{l}}, \quad l \in \mathbf{Z}
$$

Now we are ready to prove the Theorem stated in the introduction.

Proof of the Theorem. Using the operator $\hat{\mathcal{Z}}_{l}$ from expression (5) and Conditions (1) and (2), we rewrite system (1) in the following way:

$$
\sum_{l=1}^{T} \hat{\mathcal{Z}}_{m-l} I_{l}=E_{m}, \quad m=1, \ldots, T .
$$

Let us prove (iii) first. Let $\hat{\mathcal{Z}}$ be the $T \times T$ matrix operator with the elements $\hat{\mathcal{Z}}_{m-l}$ where $m, l=1, \ldots, T$. Then system (13) becomes

$$
\hat{\mathcal{Z}} I=E,
$$

where $I$ and $E$ are $T$-dimensional vector functions with the elements $I_{l}$ and $E_{m}$, respectively. According to the Corollary and to reference [1],$\hat{\mathcal{Z}}$ is a matrix classic elliptic PDO of order 1 in the Sobolev scale of $T$-dimensional complex vector functions on $S$. Therefore all solutions $I_{l}$ of system (13) belong to $H_{t+1}$ for every $l=1, \ldots, T$.

Now let us prove (i). The action of the $T \times T$ matrix operator $\hat{\mathcal{Z}}$ on the $T$-dimensional vector function $I$ given by the lefthand side of system (13) can be viewed as a discrete convolution over variables $m$ 
and $l$. Diagonalizing the matrix operator $\hat{\mathcal{Z}}$ by applying the discrete Fourier transform to both sides of system (13), we arrive at system (6), where functions $I_{s}$ are given by the discrete Fourier transform of $I_{l}$ :

$$
I_{s}=\sum_{l=1}^{T} e^{-i(2 \pi / T) s l} I_{l} .
$$

Let us comment that use of the discrete Fourier transform is justified since $\hat{\mathcal{Z}}_{l} \in O_{(t+1 \rightarrow t)}, E_{m} \in H_{t}, I_{l} \in H_{t+1}$ for every $m, l=1, \ldots, T$, where $O_{(t+1 \rightarrow t)}, H_{t}$ and $H_{t+1}$ are Banach spaces. Since the discrete Fourier transform is nondegenerate, we conclude that systems (13) and (6) are equivalent. The solution of system (13) and hence of system (1) can be found as the inverse discrete Fourier transform of the solution of system (6) as we stated in the Theorem.

In order to prove (ii), it is enough to notice that, according to expression (4) and to the Corollary, the operator $\hat{\mathcal{Z}}_{s}$ for every $s=$ $1, \ldots, T$, is the linear combination of $T$ operators: $\left.\hat{\mathcal{Z}}_{l}\right|_{l=T}$ which is a classic elliptic PDO of order 1 , and $\hat{\mathcal{Z}}_{l}$ with $l=1, \ldots, T-1$, which are operators of order $-\infty$.

Conclusion. Under natural conditions of periodicity and nonresonance we reduce the infinite system of (1) of integrodifferential equations to a finite system (6) of independent pseudodifferential equations on the unit circle with classic elliptic pseudodifferential operators of order 1 in the Sobolev scale. System (1) determines electric currents on the infinite network $\Gamma$ of nonintersecting infinitely smooth simple closed curves in the plane obtained from a fixed curve by parallel translation by vectors belonging to a one-dimensional lattice. An incident electromagnetic field is assumed to be harmonically time dependent and to be normally polarized so that the electric currents flow only along the curves. The medium is assumed to be linear and isotropic. The problem of determining such electric currents arises in numerous applications in radio physics and electrical engineering. Another significant outcome of this work is that this reduction allows one to apply the many known powerful methods for the numerical analysis of classic elliptic pseudodifferential equations on the unit circle to the original system (1).

The result presented in this paper can be generalized easily to the case of a finite number $N$ of layers of networks $\Gamma$ with, generally speaking, 
different curves for each layer. In this case, the operators $\hat{\mathcal{Z}}_{s}$ of system (6) are $N \times N$ matrix classic elliptic pseudodifferential operators of order 1 in the Sobolev scale of $N$-dimensional complex vector functions on the unit circle. Also we point out that the periodicity condition may be dropped from the Theorem. In this case system (6) becomes a one-parameter family of pseudodifferential equations whose properties we will investigate in the forthcoming papers.

Acknowledgments. I would like to thank my wife Svetlana for typing the manuscript, and Drs. Anne Dow and Cathy Gorini and also Jack Engstrom for their help in editing the manuscript.

\section{APPENDIX}

Throughout the Appendix we shall use the terminology and notation introduced in the paper.

The boundary condition for the electromagnetic field in the case under consideration has the following form [10]

$$
I_{m}(\xi)=\sigma(\xi) E_{m}^{\text {tot }}(\xi), \quad m \in \mathbf{Z}, \xi \in S,
$$

where $E_{m}^{\text {tot }}(\xi)$ is the complex amplitude of the tangential component of the total electric field at the point $\xi$ on curve $\gamma_{m}$ in $\Gamma$.

Using the superposition principle for the electromagnetic field we obtain the following expression for $E_{m}^{\text {tot }}$ :

$$
E_{m}^{\mathrm{tot}}(\xi)=E_{m}(\xi)+\sum_{l=-\infty}^{\infty} E_{m, l}(\xi),
$$

where $E_{m}(\xi)$ is the complex amplitude of the tangential component of the incident electric field at the point $\xi$ on curve $\gamma_{m}$ and $E_{m, l}(\xi)$ is the complex amplitude of the tangential component of the electric field at the point $\xi$ on curve $\gamma_{m}$ induced by the complex amplitude of the current density $I_{l}$ along curve $\gamma_{l}$.

In order to find $E_{m, l}(\xi)$ we express it [10] in terms of scalar and vector potentials as

$$
E_{m, l}(\xi)=-\frac{d}{d \xi} \Phi_{m, l}(\xi)-\frac{i \omega}{c} A_{m, l}(\xi),
$$


where $\Phi_{m, l}(\xi)$ and $A_{m, l}(\xi)$ are the complex amplitudes of the scalar potential and of the tangential component of the vector potential, respectively, at the point $\xi$ on curve $\gamma_{m}$ induced by the complex amplitude of the current density $I_{l}$ along curve $\gamma_{l}$. With the help of Green's function for the Helmholtz equation in the plane with radiation condition at infinity, these scalar and the vector potentials can be expressed in terms of the complex amplitudes of the current density $I_{l}$ and of the charge density $q_{l}$ on curve $\gamma_{l}$ :

$$
\begin{aligned}
\Phi_{m, l}(\xi) & =\frac{1}{\varepsilon} \frac{i}{4} \int_{0}^{2 \pi} H_{0}^{(2)}(k|\bar{x}(\xi)-\bar{x}(\eta)+\bar{h}(m-l)|) q_{l}(\eta) d \eta \\
A_{m, l}(\xi) & =\frac{\mu}{c} \frac{i}{4} \int_{0}^{2 \pi}\langle\tau(\xi) ; \tau(\eta)\rangle H_{0}^{(2)}(k \mid \bar{x}(\xi) \\
& -\bar{x}(\eta)+\bar{h}(m-l) \mid) I_{l}(\eta) d \eta
\end{aligned}
$$

Using the continuity equation, which in our case takes the form $q_{l}(\xi)=-(1 / i \omega)(d / d \xi) I_{l}(\xi)$, together with the definitions of the operators $\hat{L}_{m-l}$ and $\left(\hat{1} / C_{m-l}\right)$ from expression (3), and using expression (A.4), we rewrite the relation (A.3) as follows:

$$
E_{m, l}(\xi)=-\frac{i \omega}{c^{2}}\left(\hat{L}_{m-l} I_{l}\right)(\xi)-\frac{1}{i \omega}\left(\frac{\hat{1}}{C_{m-l}} I_{l}\right)(\xi)
$$

Substituting the above expression into (A.2), we rewrite the boundary condition for the electromagnetic field (A.1) as follows

$$
\begin{aligned}
I_{m}(\xi)=\sigma(\xi)\left(E_{m}(\xi)+\right. & \sum_{l=-\infty}^{\infty}\left(-\frac{i \omega}{c^{2}}\left(\hat{L}_{m-l} I_{l}\right)(\xi)\right. \\
& \left.\left.-\frac{1}{i \omega}\left(\frac{\hat{1}}{C_{m-l}} I_{l}\right)(\xi)\right)\right), \quad m \in \mathbf{Z}, \xi \in S .
\end{aligned}
$$

Dividing both sides of the above expression by $\sigma(\xi)$ and taking into account the definitions of the operators $\hat{Z}_{m-l}$ and $\hat{R}_{m-l}$ from expressions (2) and (3), we arrive at the desired system (1).

In the case of perfect conductors, the boundary condition for the electromagnetic field (A.1) has to be replaced $[\mathbf{1 0}]$ by $E_{m}^{\text {tot }}(\xi)=0$ for all $m$ in $\mathbf{Z}$ and $\xi$ in $S$, again yielding the desired system (1), but now with $\hat{R}_{m-l}$ being identically zero operators. 


\section{REFERENCES}

1. M.S. Agranovich, Spectral properties of diffraction problems, in The generalized method of eigenvibrations in diffraction theory (N.N. Voytovich, B.Z. Katsenelenbaum and A.N. Sivov, eds.), Nauka, Moscow, 1977.

2. - On elliptic pseudodifferential operators on a closed curve, Trans. Moscow Math. Soc. 47 (1984), 22-67.

3. M.S. Agranovich and B.A. Amosov, On matrix elliptic pseudodifferential operators on a closed curve, Func. Anal. Appl. 15 (1981), 79-81.

4. B.A. Amosov, An approximate solution of an elliptic pseudodifferential equation on a smooth closed curve, Achieve. Math. Sciences 40 (1985), 215-216.

5. - On an algorithm for an approximate solution of an integral equation of diffraction theory, Radio Engineer. Elec. 32 (1987), 490-497.

6. - Application of the approximate spectral method to the numerical analysis of an integral equation of diffraction theory on a smooth closed curve, Radio Engineer. Elec. 30 (1985), 1063-1070.

7. V.A. Kholodnyi, Reduction of an infinite system of integral equations of potential type on a one-dimensional lattice of closed curves in the plane to a finite system of independent pseudodifferential equations on a circle, J. Integral Equations Appl. 7 (1995), 37-46.

8. G.A. Korn and T.M. Korn, Mathematical handbook, McGraw-Hill, New York, 1986.

9. U. Lamp, K.-T. Schleicher and W.L. Wendland, The fast Fourier transform and the numerical solution of one-dimensional boundary integral equations, Numer. Math. 47 (1985), 15-38.

10. L.D. Landau and E.M. Lifshitz, Electrodynamics of continuous media, Pergamon Press, New York, 1984.

11. E.I. Nefedov and A.N. Sivov, Electrodynamics of periodic structures, Nauka, Moscow, 1966.

12. R.A. Silin and V.P. Sazonov, Slow wave systems, Sov. Radio, Moscow, 1966.

Physics Department, Maharishi International University, $1000 \mathrm{~N}$. 4th St., DB 1069, FAirfield, IA 52557-1069 